\title{
Mechanisms of hydrodynamic instability in concentration polarization
}

\author{
P. Kumar $\odot^{*}$ \\ Ashoka University, Rajiv Gandhi Education City, Sonepat, Haryana-131029, India \\ S. M. Rubinstein ${ }^{\dagger}$ \\ School of Engineering and Applied Sciences, Harvard University, Cambridge, Massachusetts 02138, USA \\ I. Rubinstein ${ }^{\ddagger}$ and B. Zaltzman ${ }^{\S}$ \\ Blaustein Institutes for Desert Research, Ben-Gurion University of the Negev, Sede Boqer Campus 8499000, Israel
}

(Received 24 May 2020; accepted 14 August 2020; published 3 September 2020)

\begin{abstract}
One-dimensional steady-state passage of direct electric current from a binary electrolyte solution into a chargeselective solid such as a metal electrode or an ion exchange membrane is hydrodynamically unstable. Instability is preceded by concentration polarization, i.e., depletion of the electrolyte in the interface diffusion layer, and it yields a microvortical flow in this layer. An ambiguity persists regarding the mechanism of this instability. The buoyant mechanisms are disregarded because instability also occurs in a gravitationally stable position and in diffusion layers too thin for buoyancy to mediate the flow. Therefore, instability is attributed to the electric forces acting in or near the interface electric double layer. These forces cause a sliplike flow known as electro-osmosis, which comes in two varieties. One is the classical equilibrium electro-osmosis related to the space charge of the electric double layer. The other is the nonequilibrium electro-osmosis related to the extended space charge that forms near the interface at high depletion. Both types of electro-osmosis may yield instability. The question is which one is at work in each particular system. The nonequilibrium electro-osmotic instability, unlike the equilibrium one, is of the short-wave type. This implies that its induced vortices are small compared to the width of the diffusion layer. Therefore, this width, which has not been clearly defined in most experiments so far, is crucial for the identification of the instability mechanism. In this paper, we report the results of our combined experimental and theoretical study of concentration polarization in a custom-designed experimental cell with a particular cation exchange membrane. As a part of our study, we investigate the recently predicted thermoelectroconvective instability. This instability is of the long-wave type and its related flow involves a pair of wide vortices spanning the diffusion layer. We experimentally retrieve this flow, which clearly marks the width of the diffusion layer. We observe that for high voltages this thermoelectroconvection is accompanied by electro-osmotic instability. Upon the background set by thermoelectroconvection, we are able to conclude that the observed electro-osmotic instability is of the short-wave type and is thus due to the nonequilibrium electro-osmosis. We suggest that a similar approach might be useful for identifying the instability mechanism in other charge-selective systems as well.
\end{abstract}

DOI: 10.1103/PhysRevResearch.2.033365

\section{INTRODUCTION}

One-dimensional steady-state passage of the direct electric current from an aqueous binary electrolyte solution into a charge-selective (perm-selective) solid is a fundamental electrochemical transport situation and a cornerstone of numerous

\footnotetext{
*pramoda.kumar@ashoka.edu.in

†shmuel@seas.harvard.edu

†robinst@bgu.ac.il

§boris@bgu.ac.il
}

Published by the American Physical Society under the terms of the Creative Commons Attribution 4.0 International license. Further distribution of this work must maintain attribution to the author(s) and the published article's title, journal citation, and DOI. technological processes. Such solids include metal electrodes, ion exchange membranes, and micro/nanochannel junctions, which are employed in cathodic electrodeposition of metals, desalination of saline water by electrodialysis, and preconcentration of proteins, respectively. DC current passage through perm-selective interfaces induces variations of concentration in the few-hundred-micron-thick interface diffusion layer, that is, concentration polarization (CP). About two decades ago, it was finally established that quiescent $\mathrm{CP}$ is hydrodynamically unstable, which explained the longstanding riddle of overlimiting conductance [1-10]. What remained unclear was the mechanism driving this instability. For some time, it had been attributed to the nonequilibrium electroconvection related to the extended space charge that forms at the outer edge of the electric double layer (EDL) at the limiting current [1-3,916]. This attribution followed from the fact that the original theoretical model in which the instability was predicted and 
many studies that followed thereon assumed a perfectly permselective solid with infinite conductivity; for such a solid, only the aforementioned nonequilibrium mechanism is possible.

Recently, it has been shown that for realistic perfectly perm-selective solids with finite conductivity (commercial ion-exchange membranes or metal electrodes) and nonperfectly perm-selective solids, an additional electroconvective instability mechanism not related to the extended space charge is possible $[7,8]$. In some sense, the ambiguity regarding the instability mechanism is similar to the one that existed until the mid-1960s around the question of what drives the flow in convective cells in a thin oil layer in a frying pan heated from below: the buoyancy or the temperature dependence of the surface tension, that is, the Rayleigh-Bénard or the Marangoni instability, a conundrum that in the end was resolved in favor of the latter.

As for the instability under consideration, it was recognized that in most practical situations for low concentration electrolytes (below one centimolar) the buoyancy mechanisms cannot yield instability [17-20]. The density stratification in the diffusion layer resulting from $\mathrm{CP}$ is due to direct concentration variation and its related nonuniform Joule heating. The temperature variations related to the latter usually do not exceed a tenth of a degree Kelvin. As a result, the Rayleigh number related to the thermally induced density stratification is much smaller than that related to concentration variation. The latter, in turn, is lower than the Rayleigh-Bénard instability threshold for unstable orientation (with a depleted interface at the bottom). For this reason, the electroconvective mechanisms had been invoked to explain the instability, and no systematic study of the Joule heating effects in CP had been undertaken until recently.

This shortcoming was remedied in the recent theoretical studies by Demekhin et al. [21]. They discovered a very interesting possibility of a Joule-heating-related thermoelectroconvective instability expected to occur for an overall stable density stratification with a depleted interface on the top. In this paper, we report the results of our combined experimental and theoretical study of the aforementioned instabilities and their contribution to overlimiting conductance in $\mathrm{CP}$ for a custom-designed experimental cell and a particular cation exchange membrane. For the beginning, let us qualitatively elucidate the physical mechanisms behind these instabilities by tracing the system's response to an accidental test vortex [3]; see Fig. 1. In the case of instability, the vortex size is identified as the critical wavelength scale of the perturbation. Vortex acceleration is identified as a positive feedback and a potential source of instability, whereas deceleration is viewed as evidence of negative feedback and stability. Generally, two characteristic times are at play. The first is the vortex lifetime defined by the vortex size divided by its typical velocity. This velocity is estimated from the buoyant and viscous force balance for gravitational mechanisms and from the slip velocity for electro-osmotic ones. The second characteristic time is that of diffusional relaxation of the temperature or concentration perturbation induced by the flow. When the relaxation time is equal to or longer than the vortex lifetime and the feedback is positive, instability is possible. In what follows, we discuss the particular instabilities that are the novel thermoelectroconvective instability, the classical Rayleigh-Bénard instability, and

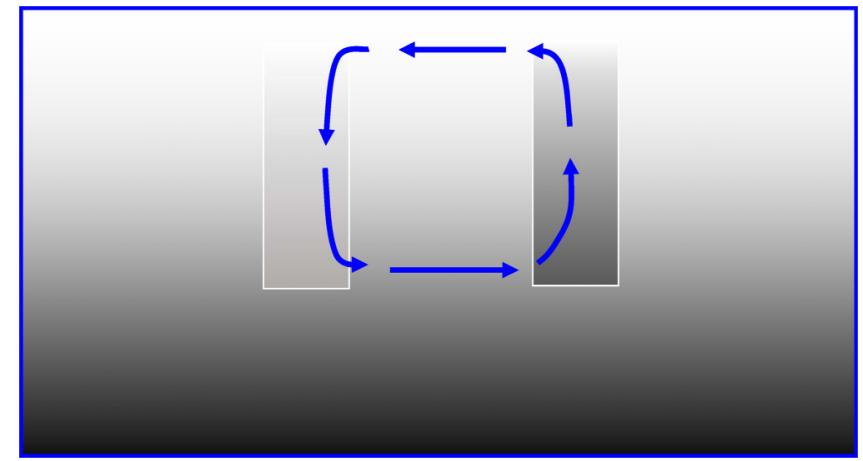

FIG. 1. Schematic representation of the thermoelectroconvective instability mechanism. Darker color corresponds to higher conductivity; blue line, streamline of the test vortex.

the two electro-osmotic instabilities, i.e., the equilibrium and the nonequilibrium one.

For the thermoelectroconvective instability, the test vortex is superimposed upon a diffusion layer operated at constant voltage with conductivity stratified by $\mathrm{CP}$. A concentrated high conductivity solution is on the bottom (anode) and depleted low concentration is on the top (cathode). The concentration perturbation by the part of this vortex ascending toward the depleted interface produces a low-resistance fluid column, whereas the descending part produces a high-resistance one. The Joule heating, proportional to the voltage squared divided by resistance is thus increased in the ascending column, yielding thermal expansion, and reduced in the descending column, resulting in thermal compression. This yields a positive feedback without diffusion relaxation, since Joule heating operates permanently, and the vortex-induced heat production in each fluid column persists with the vortex itself; thus the temperature perturbation is not dissolved by the heat diffusion in the vortex plane but only mitigated by it. The increase of the vortex size reduces the stabilizing effect of heat diffusion, which alludes to the possibility of long-wave instability. This stands in contrast with the Rayleigh-Bénard instability where the initial temperature perturbation induced by the test vortex is dissolved by heat diffusion in the vortex plane. As a result, the diffusion layer thickness is singled out as the length scale for the critical wavelength of the Rayleigh-Bénard instability. This scaling is also valid for the equilibrium electro-osmotic instability unlike the nonequilibrium one. For the nonequilibrium electro-osmosis, the slip velocity is proportional to the tangential variation of the logarithm of the normal derivative of the concentration, [1]. This is in fact diffusio-osmosis, with slip velocity proportional to the tangential derivative of the concentration logarithm, asymptotically adjusted for the singular limit of the vanishing interface concentration. In this limit, keeping the leading-order term in the power expansion of the interface electrolyte concentration and replacing the tangential variation of the concentration logarithm by that of its normal derivative, one arrives at the expression for the nonequilibrium electro-osmosis slip velocity. We apply this expression to estimate the test vortex lifetime and find that it is proportional to the third power of the perturbation wavelength, that is, the vortex size. This vortex lifetime is to be compared with the characteristic time for the diffusion 


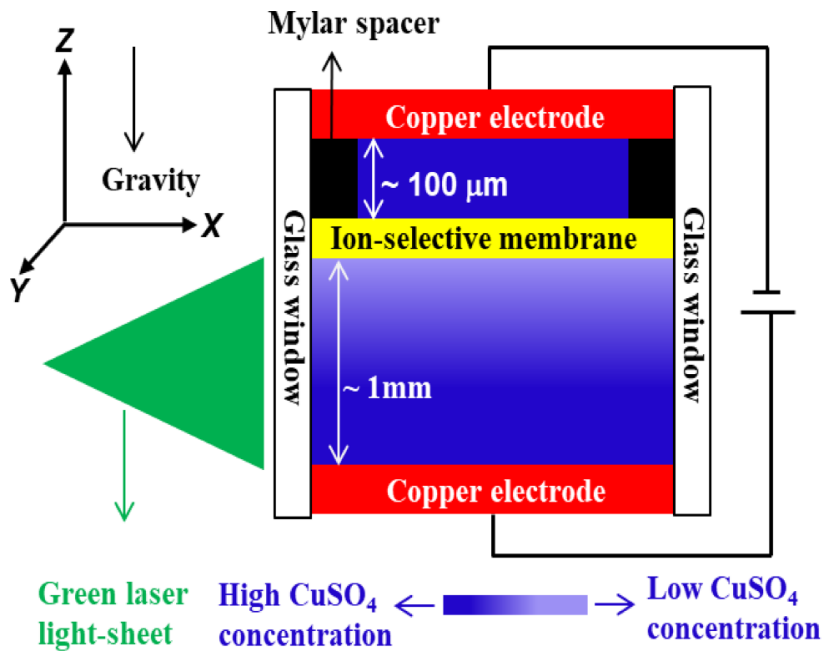

(a)

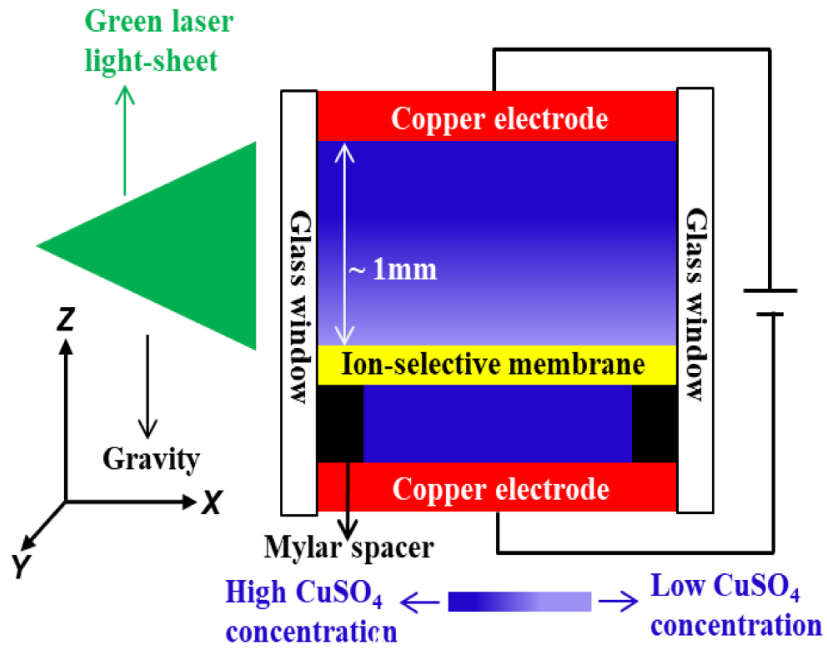

(b)

FIG. 2. Schematic side cross section of the measurement cell. The scale separation of two compartments is chosen in such a way that, for an applied potential difference, the entire $\mathrm{CP}$ occurs between the anode and the membrane, and it is schematically represented by a gradient in blue, with dark blue indicating higher concentration of the electrolyte; note that due to the small width $(\sim 100 \mu \mathrm{m})$ of the cathodic compartment, there is practically no $\mathrm{CP}$ between the membrane and the cathode. The mass-density stratification due to the $\mathrm{CP}$ is gravitationally stable in (a) and unstable in (b).

relaxation, which is of the order of wavelength squared. Since for the short wavelength the quadratic dependence dominates over the cubic one, we conclude that small vortices survive diffusion relaxation. This is the essence of the shortwavelength character of the nonequilibrium electro-osmotic instability and its main signature. The small vortices induced by the instability fuse and grow in size, which determines the subcriticality of this instability and its related hysteresis in the steady state $[1,4,7]$. As we show in this paper, an efficient gravitational stabilization in a thick diffusion layer precludes this vortex evolution and preserves the shortwave character of the overlimiting conductance as a major signature of the nonequilibrium mechanism. Since our purpose is to clarify the mechanism of instability, we focus on the vicinity of the transition. In this, we differ from the studies by de Valença et al. $[19,20]$, which investigate the current range far above the transition.

Upon this qualitative introduction, we report our experimental results followed by a theoretical analysis and discussion. Some experimental and theoretical details are diverted to the Appendixes.

\section{EXPERIMENTS}

A schematic side-view of the experimental cell is given in Fig. 2(a). This is an upright cell constructed from two identical parts, with the bottom part having glass windows for visualization from the sides. A three-dimensional drawing of the cell and other details are given in Appendix A. The electrolyte solution was prepared by dissolving copper sulfate pentahydrate powder from Sigma-Aldrich (98\% pure) in double distilled water from Baker. A $10 \mathrm{mM}$ concentration copper sulfate electrolyte solution was used in this study, and
$1 \mu \mathrm{m}$ green fluorescent microspheres (from Fisher Scientific) were mixed in the electrolyte solution in a very low concentration (100 particles $/ \mathrm{mL})$ as a tracer for flow visualization. A 150- $\mu$ m-thick cation-exchange membrane (Neosepta CMX, Tokuyama Soda, Japan) was placed next to the cathode with a $100 \mu \mathrm{m}$ spacer between them; the membrane had been soaked in the electrolyte solution for about $48 \mathrm{~h}$ before the experiment. The experimental cell was placed in front of a homemade horizontal microscope equipped with a long working distance objective from Mitutoyo to visualize the flow. The experimental cell was illuminated by a green laser light sheet employed perpendicular to the optical path of the microscope, and the image formed by the scattered fluorescent light was captured on a Raptor photonics camera (OS4MPc-CL) attached to the microscope. The images were processed using the IMAGEJ software. A Keithley source meter 2401, controlled through a computer by a MATLAB program, was connected across the cell to apply the electric potential difference.

Under the influence of a DC current, copper ions dissolve from the anode and pass through the anodic diffusion layer, the membrane, and the cathodic diffusion layer to be reduced at the cathode. Because the conductivity is much higher in the electrodes and the membrane relative to the solution, in the absence of motion, the electric field in the anodic and cathodic compartments is directed strictly upward, and is independent of the lateral coordinate. Correspondingly, for the gravitationally stable stratification, the electrolyte concentration varies only in the vertical direction in the bottom 'thick' compartment, increasing at the anode and dropping at the membrane. As a result, a purely one-dimensional (1D) conduction state develops in this compartment with the electrolyte concentration approaching a linear distribution with 

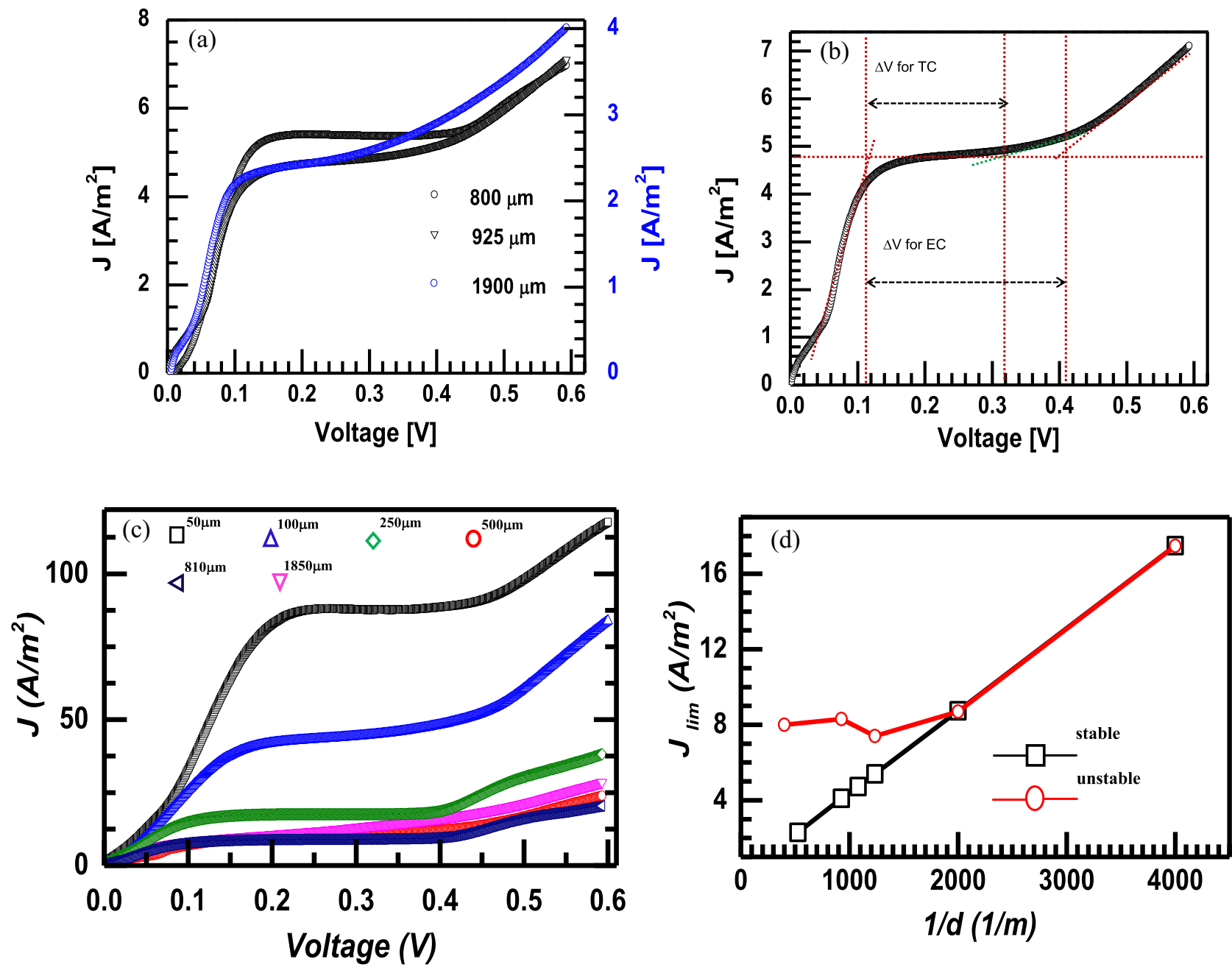

FIG. 3. (a) The current-voltage measurements for a $10 \mathrm{mM}$ copper sulfate solution at different cell gaps $(800 \mu \mathrm{m}, 925 \mu \mathrm{m}$, black scale; $1900 \mu \mathrm{m}$, blue scale). Up to around $800 \mu \mathrm{m}$ cell gap, the $I-V$ curve has three distinct regimes (Ohmic, diffusion-limited plateau, and overlimiting regimes) with a well-defined plateau width. Above this cell gap, the plateau width shrinks and turns smoothly to the overlimiting regime. Part (b) shows how we defined thresholds for thermoelectroconvection and electroconvection. (c) The current-voltage dependence for unstable cell orientation (anode on top); for this gravitationally unstable orientation, a Rayleigh-Bénard convection develops in cells with an above $500 \mu \mathrm{m}$ gap, and the limiting current saturates. (d) The linear relationship between the limiting current and the inverse of the cell gap for the gravitationally stable (cathode on top) and unstable (anode on top) cell orientation; in the unstable configuration, the limiting current saturates and becomes independent of the cell gap above $500 \mu \mathrm{m}$.

a slope proportional to the magnitude of the electric current [Figs. 2(a) and 2(b)]. Due to the small width of the cathodic compartment, the effect of $\mathrm{CP}$ is negligible in it.

A typical current-voltage curve for gravitationally stable stratification is presented in Fig. 3(a) for three values of the cell gap. Voltage is increased continuously at a very small scan rate, typically $5 \mathrm{mV} / 10 \mathrm{~s}$ for a $0.1-\mathrm{cm}$-thick cell, up to $0.6 \mathrm{~V}$. Three regions are distinguishable in this curve: (i) the Ohmic low (underlimiting) current region, followed by (ii) saturation of the curve at the "limiting current" caused by the diffusion limitation of ionic transport, and (iii) an inflection and transition to the overlimiting region. These three regions are typical of most current-voltage curves of ion-exchange membranes [22]. The overlimiting region in the current-voltage curve is split into two parts for the cell gap exceeding a certain threshold of the order of $1 \mathrm{~mm}(800 \mu \mathrm{m}$ for $10 \mathrm{mM}$ electrolyte concentration), as shown in Fig. 3(b). The first part with a relatively low slope manifests a transition from the current saturation to the overlimiting conductance and is dominated by thermoelectroconvection. Upon further increase of the voltage, this part is followed by a higher slope overlimiting current regime mediated by the electroconvective flow. The same current-voltage curve for gravitationally unstable stratification is presented in Fig. 3(c) for the voltage changing in the same range. The fine structure of this curve is similar to that for the case of gravitationally stable stratification except that the overlimiting part is solely mediated by the convection arising due to electro-osmotic instability. Finally, in Fig. 3(d) we present the dependence of the limiting current on the cell's gap. Whereas in the gravitationally 

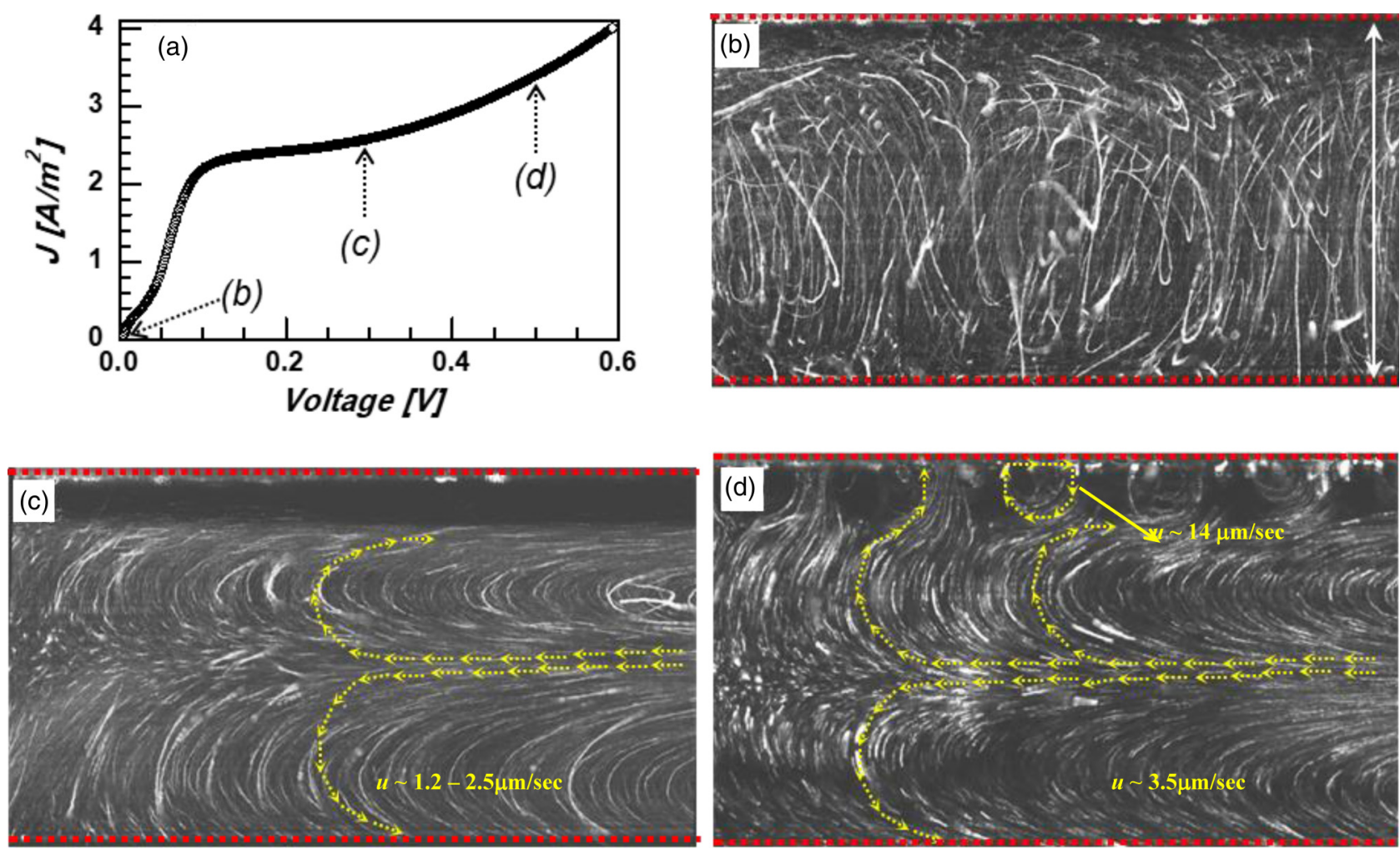

FIG. 4. Time-lapse snapshots of the flow in the cell at different voltages. $1 \mu \mathrm{m}$ fluorescent particles are uniformly dispersed in a $10 \mathrm{mM}$ copper sulfate solution to visualize the flow, and these particles are excited by a laser light sheet. The microscope is kept orthogonal to the optical path direction so that only the emitted (and scattered) light reaches the camera. The separation between the membrane and the copper electrode is $1900 \mu \mathrm{m}$. The red dotted lines indicate electrodes/membranes. (a) The $I$ - $V$ curve corresponding to this cell thickness. (b) Without any potential difference (random diffusion motion). (c) Large-scale convective flow at $0.3 \mathrm{~V}$ and (d) small convective vortices seen near the membrane surface (cathode) at $0.5 \mathrm{~V}$.

stable setup this dependence is linear, in the gravitationally unstable case, with the cell's gap exceeding a threshold, the Rayleigh-Bénard instability initiates an electrolyte flow, and the limiting current saturates at its minimal value independent of the cell's gap, implying that the effective diffusion layer width does so also.

In gravitationally stable stratification when the cell gap exceeds 800 microns, the motion of the liquid along the walls is observed for the voltage exceeding the first, thermoconvective instability threshold [Figs. 4(a) and 4(c)]. Two very long vortices emerge. The upper vortex is the thermoconvective vortex proper, whereas the bottom one results from the suppression of the former by the stable density stratification. Upon further increase of the voltage, the transition to the electroconvection dominance manifests itself in the appearance of small and fast vortices in the vicinity of the cation-selective membrane. Their formation is accompanied by a decrease of the total resistance and a further and faster rise of the current upon voltage increase [Figs. 4(a) and 4(d)].

For the case of the gravitationally unstable stratification, the formation of large round Rayleigh-Bénard vortices occupying the whole layer is observed for the cell gap exceeding a threshold ( 500 $\mu \mathrm{m})$ [Figs. 3(c) and 5]. We have not observed electroconvective vortices for the stable stratification for high voltages; the possible explanation for this is presented below in the theoretical part of the paper.

\section{THEORETICAL MODELING}

Two different mechanisms of electroconvective instability in strong electrolytes were reported in Refs. [1-5,7,8]. One is attributed to nonequilibrium electro-osmosis related to the extended space charge that develops at the limiting current in the course of $\mathrm{CP}$ at a charge-selective interface, Refs. [1-5]. The other, recently reported in Refs. [7,8], is attributed to the well-known equilibrium electro-osmosis related to the charge of the thin EDL and is able to generate a fluid flow at an imperfectly charge-selective solid or a perfectly selective charge-selective one with finite conductivity. In what follows, we will argue that the experimental observations reported above provide direct experimental evidence that the instability is mediated by the nonequilibrium electro-osmosis for the ionexchange membranes studied. For this reason, in our modeling below we focus on this mechanism, limiting our analysis to perfectly charge-selective solids, a membrane, and an anode. In Appendix B we show that for a cell gap width of 100 microns or thicker in the gravitationally stable position, the destabilizing effect of the equilibrium slip is fully suppressed by concentration stratification. In modeling the nonequilibrium electro-osmosis, we follow the asymptotic approach of $[2,3]$. This allows us to use the local electroneutrality approximation, avoiding the need to numerically resolve the EDL and the extended space charge region unfeasible for our system. 

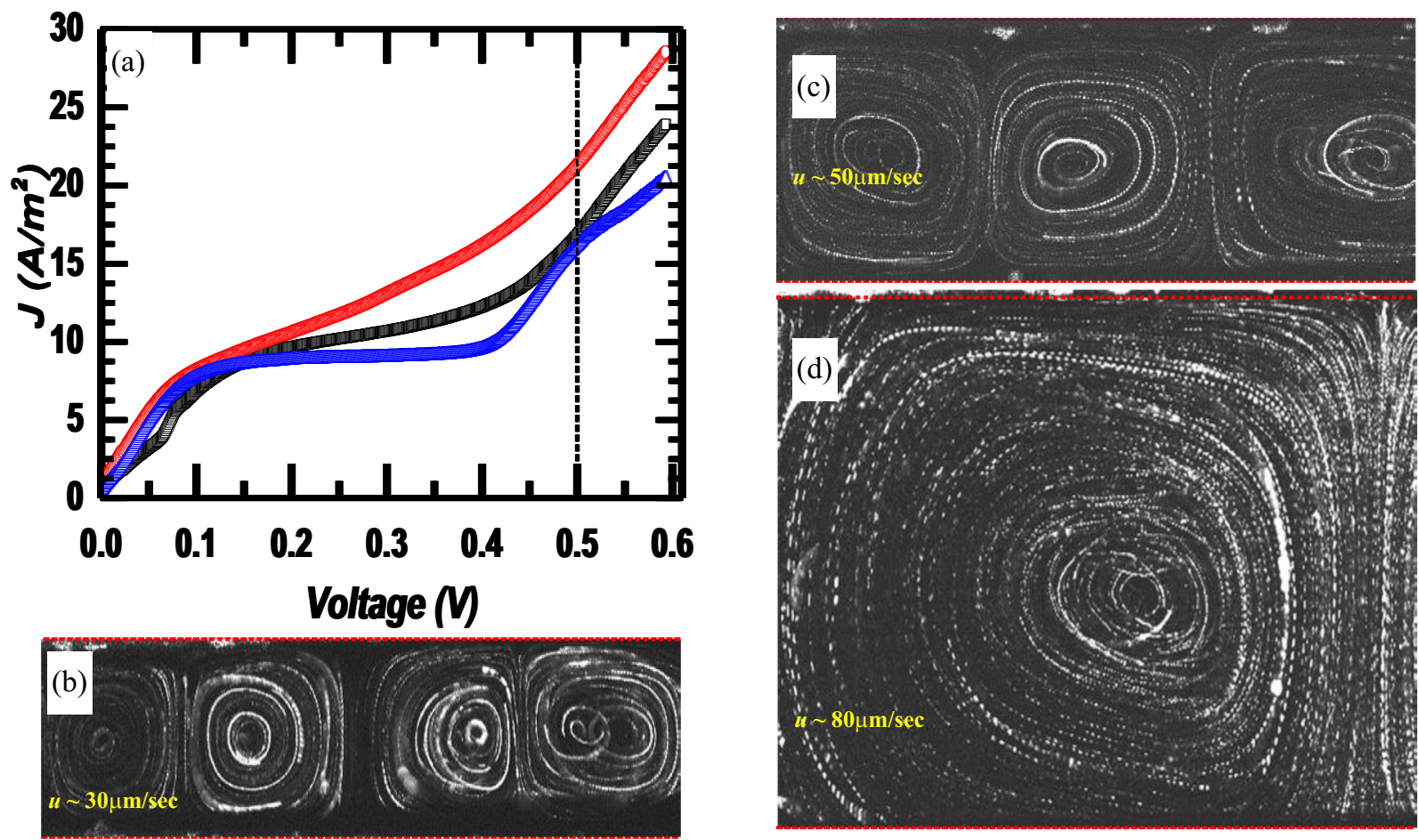

FIG. 5. (a) $I-V$ curves (a) and (b)-(d) time-lapse snapshots of the Rayleigh-Bénard convective flow in cells with the cathode at the bottom; in this configuration, the density gradient resulting from CP is gravitationally unstable. 1 micrometer size fluorescent particles are uniformly dispersed in a $10 \mathrm{mM}$ copper sulfate solution to visualize the flow; these particles are excited by a laser light sheet. The microscope is kept orthogonal to the optical path direction so that only emitted (and scattered) light reaches the camera. The red dotted lines represent the membrane (bottom) and the copper anode (top), and the separation between them is $500 \mu \mathrm{m}$ for (b), $810 \mu \mathrm{m}$ for (c), and $1850 \mu \mathrm{m}$ for (d); images are for $0.5 \mathrm{~V}$.

We consider an electrolyte layer, $0<y<1$, flanked by a cation-selective membrane, at $y=1$, and an anode, at $y=0$ (in the gravitationally unstable setup we change the orientation of the cell by redefining the dimensionless vertical variable as $y=\frac{\tilde{h}-\tilde{y}}{\tilde{h}}$ ). This one-layer system is modeled by the following dimensionless $2 \mathrm{D}$ boundary-value problem, $0<$ $y<1,-\infty<x<\infty$ :

$$
\begin{gathered}
\frac{\partial c}{\partial t}+\operatorname{Peu} \nabla c=\Delta c, \quad \nabla(c \nabla \varphi)=0 ; \\
\nabla P=\Delta \mathbf{u}+\nabla \varphi \Delta \varphi+\operatorname{Ra}_{T} T \mathbf{e}_{y}-\operatorname{Ra}_{c}(c-1) \mathbf{e}_{y}, \quad \nabla \cdot \mathbf{u}=\mathbf{0} ;
\end{gathered}
$$

$$
\operatorname{Le}\left(T_{t}+\operatorname{Peu} \nabla T\right)=\Delta T+2 c(\nabla \varphi)^{2} .
$$

Equations (1) in (1)-(3) are the electroneutral Nernst-Planck equations for the salt concentration, $c=c^{+}=c^{-}$, and the electric potential $\varphi$. Equations (2) are the Stokes and continuity equation for the dimensionless pressure $P$, velocity $\mathbf{u}$, and three dimensionless force terms on the righthand side of the Stokes equation. These are the electric Coulombic force and two buoyancy forces: the one due to temperature stratification and the other due to concentration stratification. Time derivative is absent from Eq. (2) as an approximation valid for large Schmidt numbers (kinematic viscosity to diffusivity ratio, about 1000 for the current system). Equation (3) is the convective heat equation for the dimensionless temperature, $T$, with the Joule heating term on the right-hand side. The following nondimensionalization is applied (the dimensional variables are tilded, whereas the untilded variables stand for their dimensionless counterparts): $c=\frac{\tilde{c}}{\tilde{c}_{0}}, \varphi=\frac{\tilde{\varphi}}{\tilde{\Phi}_{0}}, \quad \tilde{\Phi}_{0}=\frac{\tilde{R} \tilde{T}}{\tilde{F}}, \quad \mathbf{x}=\frac{\tilde{\mathbf{X}}}{\tilde{h}}, t=\frac{\tilde{T}}{\tilde{t}_{0}}, \quad \tilde{t}_{0}=\frac{\tilde{h}^{2}}{\tilde{D}}, \quad \mathbf{u}=$ $v \mathbf{e}_{x}+w \mathbf{e}_{y}=\frac{\tilde{\mathbf{u}}}{\tilde{v}_{0}}, \quad \tilde{v}_{0}=\frac{\tilde{D}}{\tilde{h}}, T=\frac{\tilde{T}}{\tilde{T}_{0}}, \quad \tilde{T}_{0}=\frac{\tilde{F} \tilde{D} \tilde{c}_{0}}{\tilde{a} \tilde{c}_{p} \tilde{r}_{0}} z^{2} \tilde{\Phi}, P=\frac{\tilde{P}}{\tilde{P}_{0}}$, $\tilde{P}_{0}=\frac{\tilde{\mu} \tilde{D}}{\tilde{h}^{2}}, \quad$ Le $=\frac{\tilde{D}}{\tilde{a}}, \quad \operatorname{Pe}=\frac{\tilde{\lambda}_{D} \tilde{F} \tilde{\Phi}_{0} \tilde{c}_{0}}{\tilde{\mu} \tilde{D}}, \quad \tilde{\lambda}_{D}=\sqrt{\frac{\tilde{\varepsilon} \tilde{\Phi}_{0}}{\tilde{F} \tilde{c}_{0}}}, \quad \operatorname{Ra}_{T}=$ $\frac{\tilde{\beta} g \tilde{r}_{0} \tilde{T}_{0} \tilde{h}^{3}}{\tilde{\lambda}_{D} \tilde{F} \tilde{\Phi}_{0} \tilde{c}_{0}}, \operatorname{Ra}_{c}=\frac{\tilde{g} \tilde{M}_{c u} \tilde{h}^{3}}{\tilde{\lambda}_{D} \tilde{F} \tilde{\Phi}_{0}}$. Here, $\tilde{\Phi}_{0}$ is the thermal voltage, $\tilde{h}$ is the width of the cell gap, $\tilde{D}$ is diffusivity (assumed equal for ions of both signs), $\tilde{a}$ is thermal diffusivity, $\tilde{c}_{p}$ is the specific heat capacity, $\tilde{r}_{0}$ is the density, $z$ is the valency (assumed to be the same for cations and anions), $\tilde{\mu}$ is the viscosity, Le is the Lewis number, $\mathrm{Pe}$ is the material Peclet number, $\tilde{\lambda}_{D}$ is the Debye length, $\tilde{\varepsilon}$ is the dielectric constant, $\tilde{\beta}$ is the thermal expansion coefficient, $\mathrm{Ra}_{T}$ is the temperature Rayleigh number and $\mathrm{Ra}_{c}$ is the concentration Rayleigh number, and $M_{\mathrm{Cu}}$ is the molecular weight of $\mathrm{Cu}$. We note that for a realistic physical situation and a cell gap of the order of $0.1 \mathrm{~cm}$, the parameter $\mathrm{Ra}_{T}=O(1)$ and $\mathrm{Ra}_{c}=O\left(10^{4}\right)$. For gravitationally stable stratification $\mathrm{Ra}_{T}<0, \mathrm{Ra}_{c}<0$, whereas for gravitationally unstable stratification $\mathrm{Ra}_{T}>0, \mathrm{Ra}_{c}>0$. In what follows, we consider a $10 \mathrm{mM}$ aqueous solution of the copper sulfate, and thus we set $z=2$ and use the following values for the major control parameters $\mathrm{Ra}_{T}$ and $\mathrm{Ra}_{c}: \mathrm{Ra}_{T}=$ $\pm 0.7 \times 10^{3}\left(\frac{\tilde{h}}{\mathrm{~cm}}\right)^{3}, \operatorname{Ra}_{c}= \pm 3.7 \times 10^{7}\left(\frac{\tilde{h}}{\mathrm{~cm}}\right)^{3}$. 

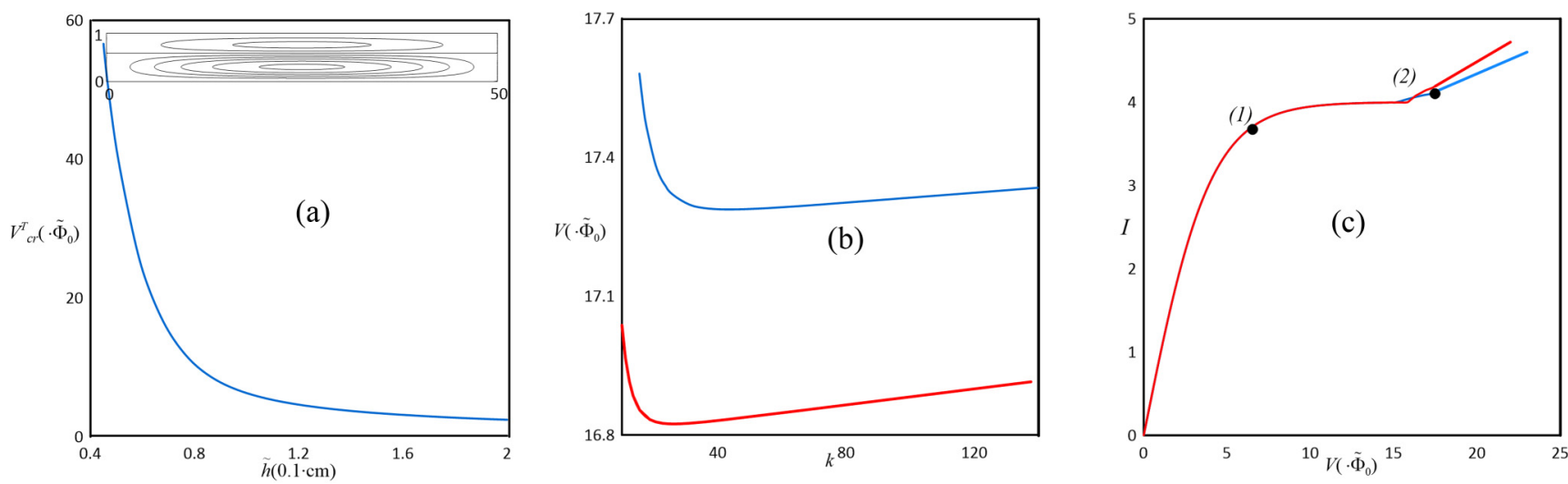

FIG. 6. (a) The dependence of the dimensionless critical voltage for thermoelectroconvective instability [point (1) on the current/voltage curve in Fig. 6(c)] on the cell gap width (instability occurs, when $V>V_{\mathrm{cr}}$ ). Inset: the level lines of the thermoelectroconvective stream function; (b) neutral stability curve for nonequilibrium electro-osmotic instability [point (2) on the current/voltage curve in (c): red line, $\tilde{h}=0.05 \mathrm{~cm}$; blue line, $\tilde{h}=0.1 \mathrm{~cm}]$; (c) dimensionless average current density, $I$; dimensionless voltage, $V$; dependence: red line, $\tilde{h}=0.05 \mathrm{~cm}$; blue line, $\tilde{h}=0.1 \mathrm{~cm}$. Point (1), the onset of the thermoelectroconvective flow; point (2), the onset of the overlimiting conductance mediated by the nonequilibrium electro-osmotic instability; $N=1000, \varepsilon=\frac{4.2 \times 10^{-7} \mathrm{~cm}}{\tilde{h}}, z=2$.

The following boundary conditions at the outer edges, $y=0,1$, of the electroneutral bulk complete the model formulation:

$y=0$ (anode/solution interface):

$$
\begin{aligned}
\left(c_{y}-z c \varphi_{y}\right) & =0, \quad T_{y}-b\left(T-T_{a}\right)=0, \\
\ln c+z \varphi & =\ln \frac{N}{z}, \quad \mathbf{u}=\mathbf{0}
\end{aligned}
$$

$y=1$ (membrane/solution interface):

$$
\begin{gathered}
\left(c_{y}-z c \varphi_{y}\right)=0, \quad T_{y}+b\left(T-T_{a}\right)=0, \quad c=\left(-\varepsilon c_{y}\right)^{\frac{2}{3}}, \\
v=-\frac{3\left(V^{*}\right)^{2}}{16}(\ln c)_{x}, \quad w=0 .
\end{gathered}
$$

In the boundary conditions (4) and (5), the first equality prescribes vanishing of the co-ion fluxes, whereas the second relation prescribes heat exchange between the cell gap and the environment. The third equality in (4) prescribes the value of the counter-ion electrochemical potential at the solution/cathode interface, whereas the fourth equality prescribes nonslip at this interface. In the boundary condition (5), $V^{*}$ is defined as $V^{*}=\ln N-z V-(\ln c+z \varphi)$ and stands for the logarithmic electrochemical potential drop across the extended space charge. The third condition in (5) stands for the relation between the salt concentration and the electric current on the outer edge of the extended space charge region in the nonequilibrium regime, Refs. [3,11-14]. The electro-osmotic slip condition in (5) stands for nonequilibrium electro-osmosis, Refs. [2,3]. Finally, $\varepsilon=\frac{\tilde{\lambda}_{D}}{\tilde{h}}$ is the dimensionless Debye length, $V$ is the dimensionless potential drop between the membrane and the cathode, and $N$ is the dimensionless fixed charge density in the membrane. For a thick diffusion layer, $\left|\mathrm{Ra}_{c}\right| \geqslant O\left(10^{2}\right)$, the equilibrium electroosmosis, as opposed to the nonequilibrium one, contributes little to the overall instability dynamics and overlimiting conductance for both gravitationally stable and unstable concen- tration stratifications (details in Appendix B). For this reason, in the model problem (1)-(5) we only address the case of the nonequilibrium slip on the membrane/solution interface and leave the discussion of the equilibrium electro-osmosis to Appendix B.

The results of the linear stability analysis and nonlinear simulation for the boundary value problem (1)-(5) for gravitationally stable orientation (membrane on the top) are presented in Figs. 6 and 7.

As mentioned before, for realistic conditions the buoyant effect of concentration stratification whose quantitative measure is the parameter $\mathrm{Ra}_{c}$ is up to four orders of magnitude stronger than that of temperature stratification whose measure is $\mathrm{Ra}_{T}$. In the linear stability analysis, the stabilizing buoyant effect of concentration stratification with $\mathrm{Ra}_{c}<0$, combined with the intense heat diffusion at short scales, manifests itself in suppressing the moderate wavelength perturbation modes resulting in a long-wave thermoelectroconvective instability in accord with the qualitative picture presented in the Introduction. Thus, for our system at the voltage slightly above the thermoelectroconvective instability threshold [Figs. 6(a) and $6(c)]$, the nonlinear unstable mode's wave number does not exceed 0.01 . This stands in radical contrast with the nonequilibrium electro-osmotic instability, which asymptotically, in the vanishing Debye length limit, exhibits the short-wave singularity, whose essence is a zero-wavelength critical unstable mode [1]. This singularity is removed by accounting for a finite Debye length, Refs. [2,3] (the asymptotic approximation employed herein), but still for a realistic Debye length the instability remains in the short-wavelength range, $k_{\mathrm{cr}} \gg 1$. This feature allows the nonequilibrium electro-osmotic instability to avoid the stabilizing effect of concentration stratification, whose efficiency decays exponentially with the increasing $k$ [Fig. 6(b)]. In the nonlinear regime this manifests itself through the relative vortex size decreasing with the increase of the cell gap width (Fig. 7).

We note that the characteristic wavelength for the equilibrium instability mechanism is of the order of unity, Refs. [7,8], 

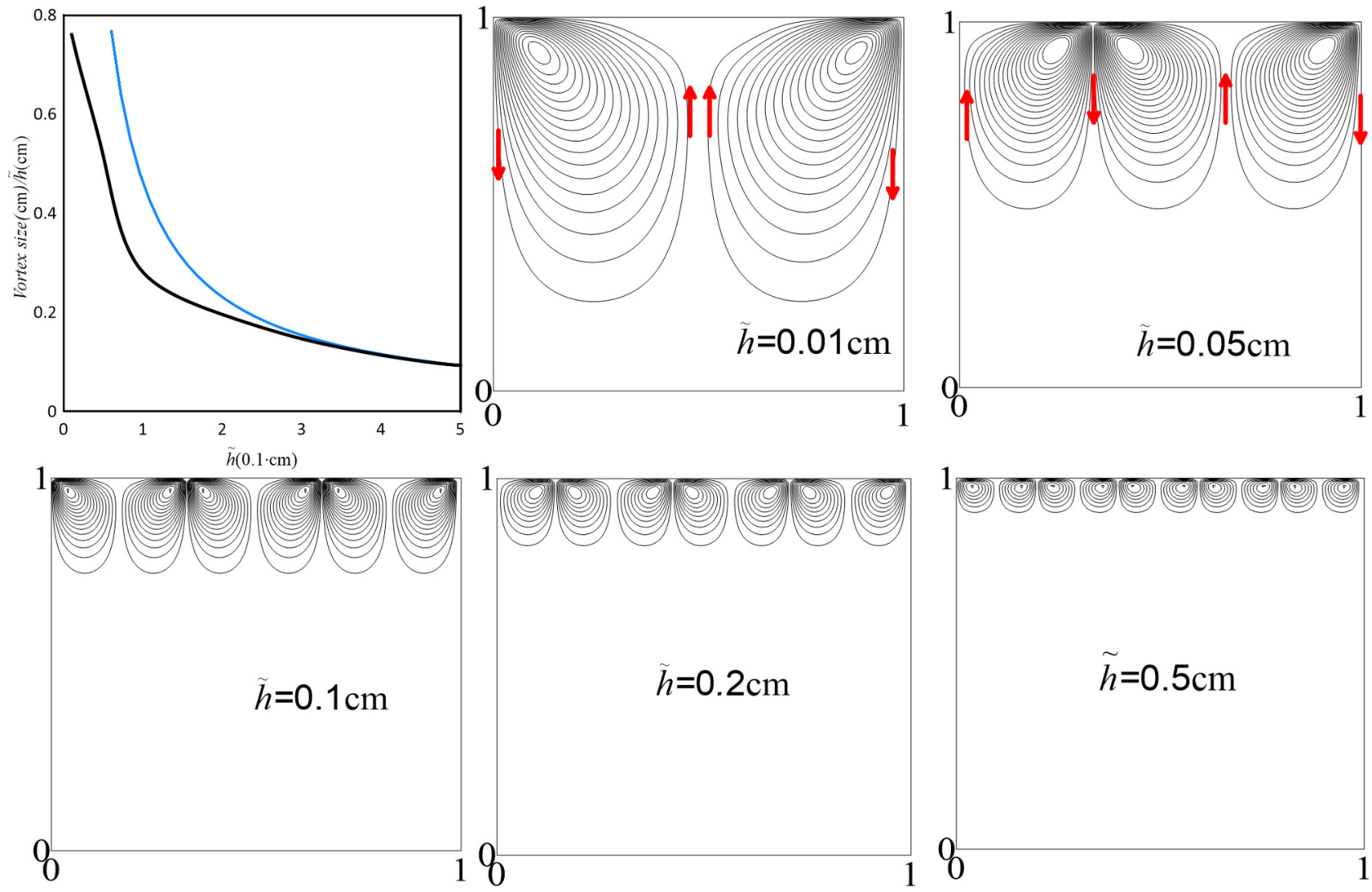

FIG. 7. The dependence of relative vortex size on the cell gap width (black line) and its approximation $\frac{0.046 \mathrm{~cm}}{\tilde{h}}$ (blue line). The corresponding stream lines' map for an increasing sequence of the cell gap widths; $N=1000, \varepsilon=\frac{4.2 \times 10^{-7} \mathrm{~cm}}{\tilde{h}}, z=2$.

and thus, for a sufficiently wide cell, instabilities of this type are fully suppressed by the stabilizing effect of concentration stratification (details are in Appendix B).

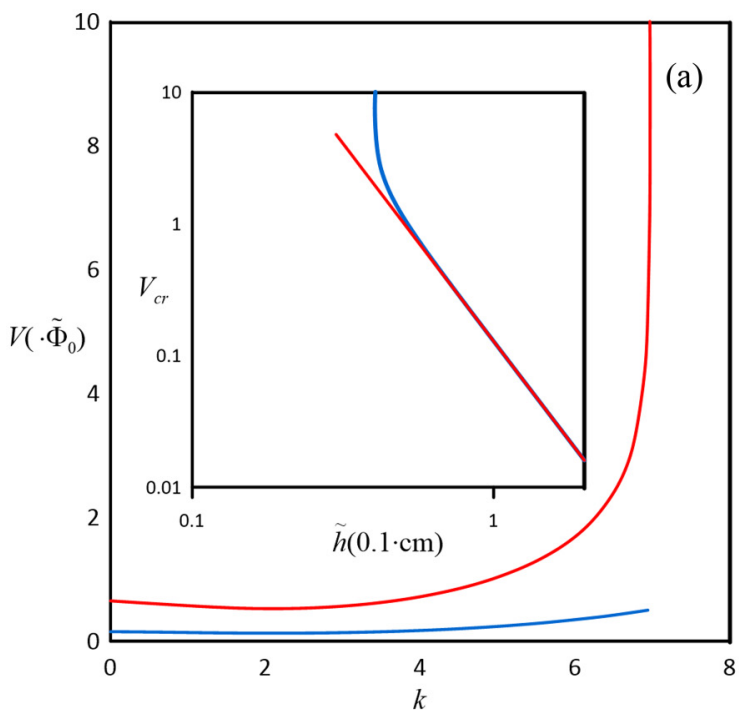

For the gravitationally unstable orientation, for the cell gap exceeding approximately half a millimeter, the RayleighBénard instability due to concentration stratification initiates

FIG. 8. (a) Marginal stability curves for $\tilde{h}=0.05 \mathrm{~cm}$ (red line) and $\tilde{h}=0.1 \mathrm{~cm}$ (blue line). Inset: the dependence of the dimensionless critical voltage on the cell gap width (blue line) and the power law- $\tilde{h}^{-3}$ approximation (red line); (b) the dependence of the dimensionless limiting current on the cell gap width (blue line) and linear approximation (red line). Inset: dimensionless average current density, $I$; dimensionless voltage, $V$; dependence for $\tilde{h}=0.05 \mathrm{~cm}$ (red line) and $\tilde{h}=0.1 \mathrm{~cm}$ (blue line); $N=1000, \varepsilon=\frac{4.2 \times 10^{-7} \mathrm{~cm}}{\tilde{h}}, z=2$. 

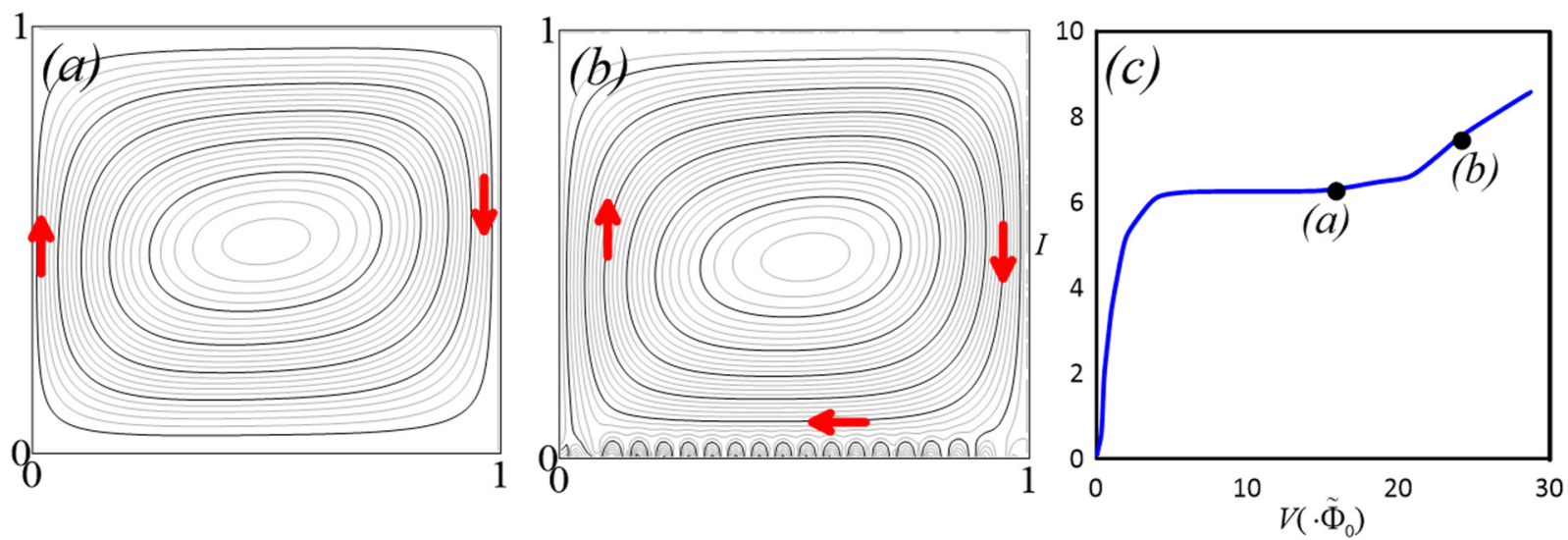

FIG. 9. Stream lines for the limiting (a) and overlimiting (b) currents; (c) the dimensionless average current density, $I$; dimensionless voltage, $V$; dependence: $\tilde{h}=0.1 \mathrm{~cm}, N=1000, \varepsilon=\frac{4.2 \times 10^{-7} \mathrm{~cm}}{\tilde{h}}, z=2$.

a fluid flow for a current below limiting (Fig. 8). The corresponding critical wave number is of the order of unity, whereas the critical voltage decreases with the increase of the cell gap width as $O\left(\tilde{h}^{-3}\right)$ [Fig. 8(a)]. As a result of the Rayleigh-Bénard convection, an effective dimensionless $O\left(\tilde{h}^{-1}\right)$-thick diffusion layer forms and so the dimensionless limiting current increases linearly with the increase of the cell gap width [Fig. 8(b)]. These results of the linear stability analysis and nonlinear simulations stand in agreement with the experimental data [Fig. 3(d)].

A further increase of voltage results in the onset of electroconvective instability and transition to overlimiting conductance (Fig. 9). In addition, for the gravitationally unstable orientation, similarly to the stable one, for sufficiently wide cells, the Rayleigh-Bénard convection suppresses the moderate wavelength modes and singles out the nonequilibrium electro-convective instability as the only possible mechanism. The resulting electroconvective vortices are confined to a very narrow vicinity of the membrane. Presumably, they were not visualized in the experiments due to their small size.

\section{CONCLUSIONS}

Electrically driven hydrodynamic instability in concentration polarization is a relatively recently discovered phenomenon that was first predicted theoretically [1] and later observed experimentally [4,5]. Definitive determination of the mechanism of this phenomenon is a fundamental challenge resolved in this study. In this sense, we did not pursue any practical implication, although the obtained results may be of direct importance for overlimiting electrodialysis [23], intensification of transport-limited electrode processes, and other applications. Motivated by this goal of identifying the mechanism of electroconvective instability, our combined experimental and theoretical study focused on the vicinity of transition near the limiting current. In this, our study differs from the previous thorough experimental studies of electroconvective instability in conjunction with buoyancy effects and without them $[19,20]$. In these studies, the authors explored the current range from three to eleven times the limiting. In this regime, for gravitationally stable orientation of the cell, both equilibrium and nonequilibrium vortices scale in size with the diffusion layer width, and thus are indistinguishable. Here, a remark is due regarding the width of the diffusion layer. The experimental studies [4,19,20,22], including the present study, employ the quiescent polarization cell. This cell was designed in [24], specifically for investigating overlimiting conductance. A particular feature of this cell is that, in contrast to the common electrodialysis cell, no desalination occurs in it, with the average solute concentration kept practically constant throughout the experiment. Moreover, the width of the diffusion layer is geometrically defined in this cell as half the distance between the copper anode and the membrane. However, this is true only for the steady state. For the voltage/current ramp conditions often used in practice, the width of the diffusion layer increases from a very small initial value, corresponding to the first vanishing of the interface concentration, to the final steadystate value. This makes it impossible to determine whether the observed growth of the emerging electroconvective vortices is due to the nonlinear evolution typical of the nonequilibrium mechanism, or is just an outcome of the thickening of the diffusion layer in accordance with the equilibrium mechanism. The only way to resolve this ambiguity is to wait for about one hour below the instability threshold until the limiting current decreases to its steady state, and only then cross the threshold to see if the emerging vortices are small compared to the diffusion layer thickness. In this respect, the thermoelectroconvective instability investigated in the current study both theoretically and experimentally provides an excellent means for clearly visualizing the diffusion layer. In the strongly overlimiting regime employed in $[19,20]$, the flow is entirely dominated by electroconvection rendering the thermoelectroconvective instability flow unobservable. Our main result thus concerned electro-osmotic instability emerging at the background of thermoelectroconvective flow mitigated by stable density stratification. The thermoelectroconvective instability was found to set a clear length scale for the width of the diffusion layer at the charge-selective interface. For a sufficiently wide cell, the stabilizing effect of density stratification suppressed the subcriticality typical for the nonequilibrium instability. This expressed itself in the elimination of the nonlinear growth of the typical vortex size preserving the short-wave character of the flow, typical 


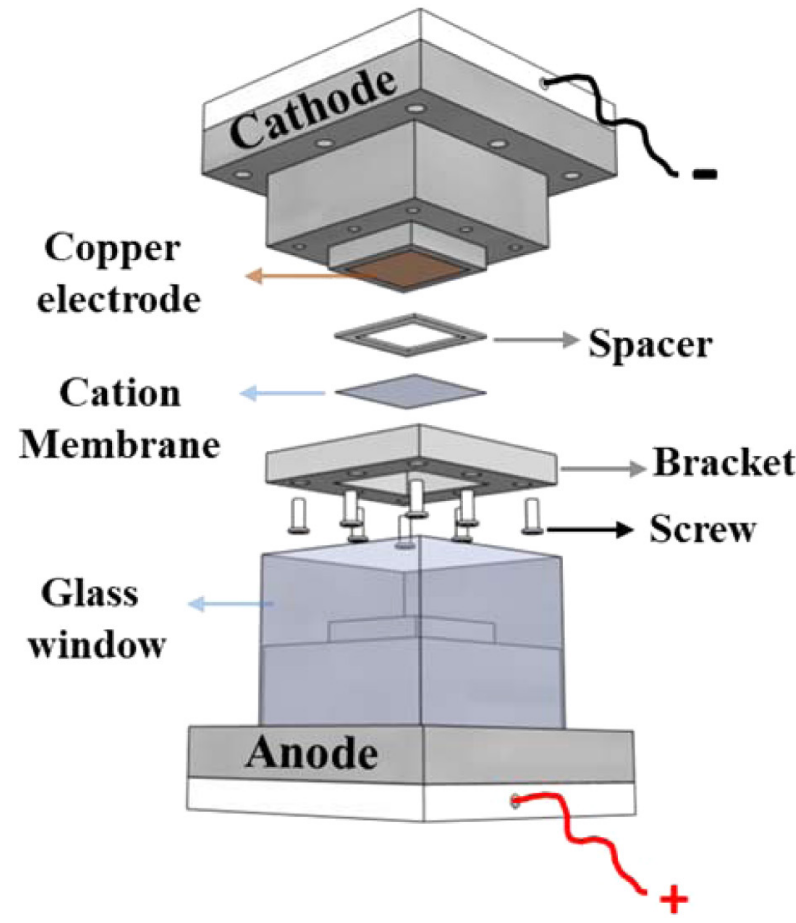

A

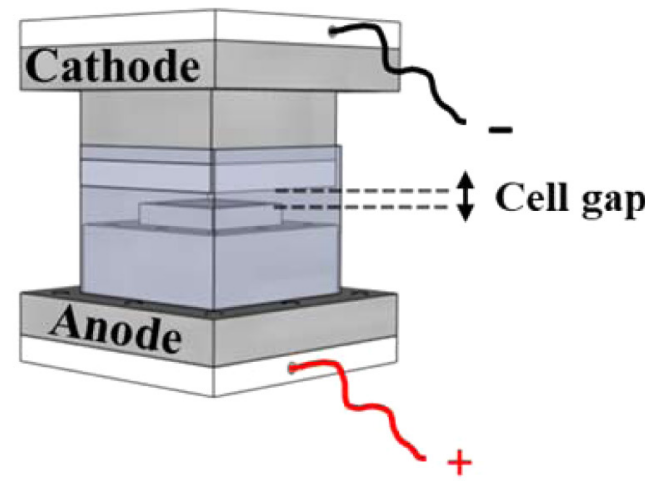

B

FIG. 10. Schematic three-dimensional drawing of the experimental electrolyte cell.

for the linear stage of the instability. Through visualization of the electroconvective and thermoelectroconvective flows, this allowed us to confidently conclude that, at least for the Neosepta CMX cation-exchange membrane addressed here, the overlimiting conductance is mediated by the nonequilibrium electro-osmotic short-wavelength instability. The shortwave character is the major robust signature of this instability that crucially distinguishes it from its equilibrium counterpart. Our experiments recover the thermoelectroconvective instability predicted theoretically [21], but never observed previously, and they confirm its long-wave character. At the same time, our modeling recovers all the novel features of the short-wave nonequilibrium electro-osmotic instability superimposed upon the long-wave thermoelectroconvective flow. This includes the two observed long-wave vortices spanning the diffusion layer, and, as a particularly remarkable feature near the transition, the arrest by gravity stabilization of the nonlinear growth of the short-wave nonequilibrium vortices for sufficiently wide cell gaps.

\section{ACKNOWLEDGMENTS}

The authors are grateful to Tatiana Philippova for editing and proofreading the manuscript, and we thank Roiy Sayag and Iris Visoly-Fisher for valuable discussions and support.

\section{APPENDIX A: THE DESCRIPTION OF THE EXPERIMENTAL CELL}

A schematic three-dimensional drawing of the experimental electrolyte cell for flow visualization is shown in Fig. 10.
This cell consists of two identical electrode parts with glass windows attached only to the bottom part (anode), as shown in Fig. 10(A). A copper block of $15 \mathrm{~mm} \times 15 \mathrm{~mm} \times 50 \mathrm{~mm}$ with a jacket machined in acrylic constitutes the electrode. A membrane is mechanically fixed by an acrylic bracket to the cathode electrode with a thin spacer $(\sim 50-100 \mu \mathrm{m})$ in between. The dimensions of the glass windows on the anode are such that the cathode part could be just slid through, as shown in Fig. 10(B). The cathode is attached to a travel translation stage with a micrometer screw to adjust the gap between the anode and the cathode accurately.

\section{APPENDIX B: EQUILIBRIUM ELECTRO-OSMOSIS IN CP IN A THICK DIFFUSION LAYER AT A PERFECTLY CHARGE-SELECTIVE INTERFACE}

In this Appendix, we show that for a thick diffusion layer, $\left|\operatorname{Ra}_{c}\right| \geqslant O\left(10^{3}\right)$, equilibrium electro-osmosis, as opposed to the nonequilibrium one, contributes little to the overall instability dynamics and overlimiting conductance for both gravitationally stable and unstable concentration stratification. We assume that the width of the cathodic compartment is negligible compared to that of the anodic one and focus on the effect of concentration stratification on the recently reported equilibrium electro-osmotic instability and its related vortical flow [8,9], neglecting the effect of thermoelectroconvection, $\mathrm{Ra}_{T}=0$. We also note that the equilibrium electro-osmotic slip occurs for moderate voltage and moderate depletion whenever the dimensionless interface concentration satisfies the inequality, $c \gg O\left(\varepsilon^{\frac{2}{3}}\right)$, and the extended space charge is absent. For a higher voltage and interface concentration of 

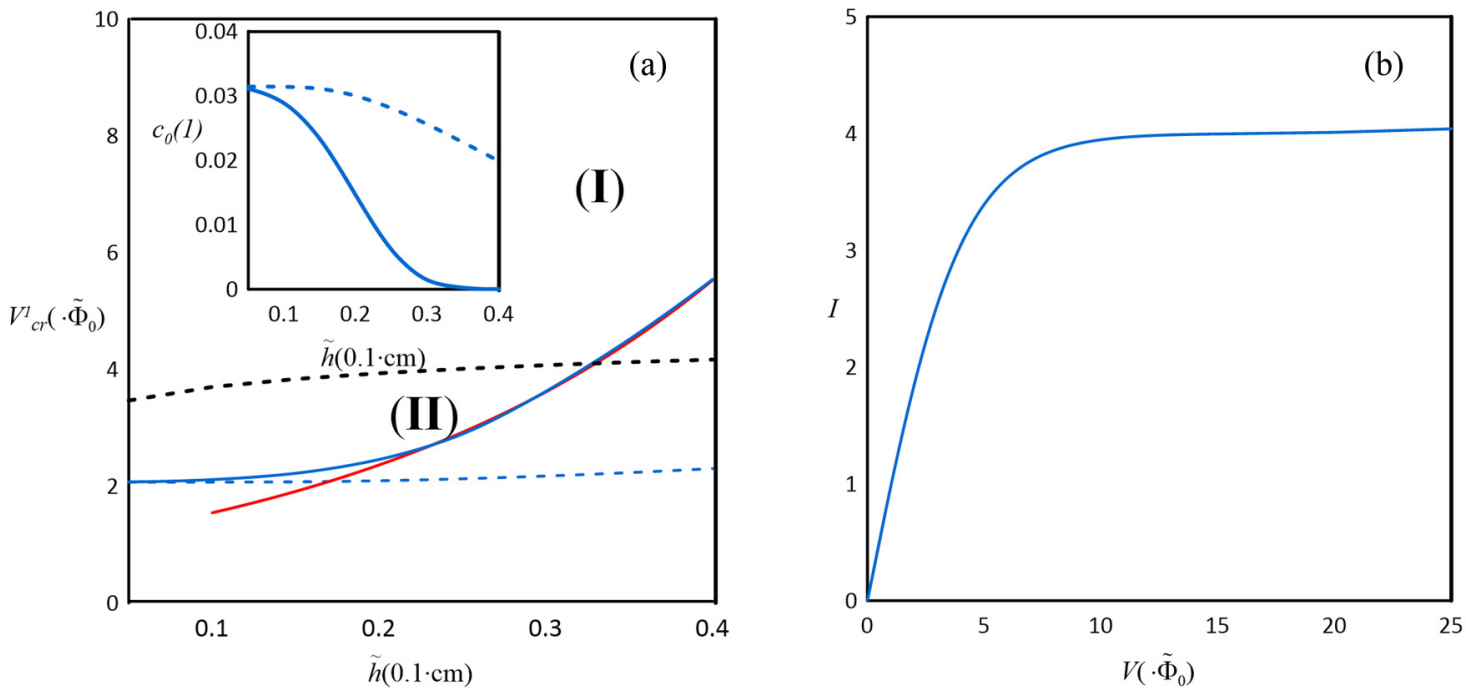

FIG. 11. (a) The dependence of the critical potential drop across the anodic compartment, $V_{\mathrm{cr}}^{1}$, on the compartment width (blue line), and its exponential approximation $e^{0.43 h}, h=\frac{\tilde{h}}{\mathrm{~cm}}\left(\right.$ red line), for gravitationally stable stratification, $\operatorname{Ra}_{T}=0, \operatorname{Ra}_{c}=-3.7 \times 10^{7}\left(\frac{\tilde{h}}{\mathrm{~cm}}\right)^{3}$ (the same dependence for $\mathrm{Ra}_{T}=0, \mathrm{Ra}_{c}=0$ is plotted by the dashed blue line). The black dashed line marks the boundary between the region of nonequilibrium EDL with the extended space charge present (I) and the region (II) of equilibrium EDL and the respective transition from the equilibrium electro-osmosis to the nonequilibrium one. Inset: the dependence of the interface concentration, $c^{0}(1)$ on the compartment width. (b) The dimensionless average current density, $I$; dimensionless voltage, $V$; dependence for the gravitationally unstable stratification, $\operatorname{Ra}_{T}=0, \operatorname{Ra}_{c}=-3.7 \times 10^{7}\left(\frac{\tilde{h}}{\mathrm{~cm}}\right)^{3}, \tilde{h}=0.1 \mathrm{~cm} . N=1000, \sigma=0.3, z=2$.

the order of $O\left(\varepsilon^{\frac{2}{3}}\right)$, the extended space charge appears, and dominates the electro-osmotic slip thereon, Eq. (5). In what follows, we address the equilibrium electro-osmosis valid for moderate voltage and the EDL preserving its equilibrium structure.

We consider the following two-layer model problem for a perfectly perm-selective cation-exchange membrane (of dimensionless thickness $\Delta$ and with fixed charge density $N$ ) and its adjacent anodic compartment (of dimensionless unity width):

Equations. For $0<y<1,-\infty<x<\infty$, Eqs. (1)-(3) hold. For $1<y<1+\Delta,-\infty<x<\infty$, the electric potential in the electroneutral bulk of the membrane, $\Phi$, satisfies Ohm's equation,

$$
\sigma \Delta \Phi=0
$$

where $\sigma$ is the dimensionless membrane conductivity. The following boundary conditions complete the formulation:

Anode/solution interface, $y=0$, conditions (4).

Membrane/solution interface, $y=1$ :

$c_{y}-z c \varphi_{y}=0, \quad T_{y}-b\left(T-T_{a}\right)=0, \quad c_{y}+z c \varphi_{y}=\sigma \Phi_{y}$,

where the last equality asserts continuity of the cationic flux (current density),

$$
\begin{aligned}
v= & (-V-\varphi)(\ln c+z \varphi)_{x} \\
& +\left(4 \ln 2-4 \ln \left[e^{\frac{-V-\varphi}{2}}+1\right]\right)(\ln c)_{x}, \\
w= & 0 .
\end{aligned}
$$

The electro-osmotic slip condition in (B3) stands for equilibrium electro-osmosis, Refs. [1,8,25].

Membrane/cathodic compartment interface, $y=1+\Delta$ :

$$
\Phi=-V
$$

The results of the linear stability analysis of the 1D steady-state solution to the problem (1)-(4) and (B1)-(B3), $c^{0}(y), \varphi^{0}(y), \Phi^{0}(y)$, for the gravitationally stable stratification, $\mathrm{Ra}_{c}<0$, are presented in Fig. 11(a). We observe a sharp exponential growth of the critical electrical potential drop across the anode compartment, $V_{\mathrm{cr}}^{1}=\varphi^{0}(1)-\varphi^{0}(0)$, with the increase of the compartment width. We also note that for the anodic compartment width exceeding $350 \mu \mathrm{m}$, the extended space charge appears, that is, the expression (B3) for the electro-osmotic slip velocity fails and should be replaced by the one for the nonequilibrium electro-osmotic slip, $v=$ $-\frac{3\left(V^{*}\right)^{2}}{16}(\ln c)_{x}$. In Fig. 11(b) we present the current/voltage dependence for the gravitationally unstable cell orientation, $\mathrm{Ra}_{c}>0$, obtained via a numerical solution of the full nonlinear problem (1)-(4) and (B1)-(B3). We observe that for the thick diffusion layer under consideration, accounting for the equilibrium electro-osmotic slip alone while disregarding the extended space charge eliminates the overlimiting conductance.

We conclude that in accord with the statement in the main text, the critical wave number for the equilibrium electroconvective instability is of the order of unity $(1<k<3)$, Refs. [8,9]; that is, for a sufficiently thick diffusion layer (with the width of the cell gap exceeding $\left.400 \mu \mathrm{m}, \mathrm{Ra}_{c}>3000\right)$ it lies in the range dominated by the stabilizing or destabilizing effect of concentration stratification. 
[1] I. Rubinstein and B. Zaltzman, Electro-osmotically induced convection at a permselective membrane, Phys. Rev. E 62, 2238 (2000).

[2] I. Rubinstein and B. Zaltzman, Wave number selection in a nonequilibrium electro-osmotic instability, Phys. Rev. E 68, 032501 (2003).

[3] B. Zaltzman and I. Rubinstein, Electro-osmotic slip and electroconvective instability, J. Fluid Mech. 579, 173 (2007).

[4] S. M. Rubinstein, G. Manukyan, A. Staicu, I. Rubinstein, B. Zaltzman, R. G. H. Lammertink, F. Mugele, and M. Wessling, Direct Observation of a Nonequilibrium Electro-Osmotic Instability, Phys. Rev. Lett. 101, 236101 (2008).

[5] G. Yossifon and H. C. Chang, Selection of Nonequilibrium Overlimiting Currents: Universal Depletion Layer Formation Dynamics and Vortex Instability, Phys. Rev. Lett. 101, 254501 (2008).

[6] H. C. Chang, G. Yossifon, and E. Demekhin, Nanoscale electrokinetics and microvortices: How microhydrodynamics affects nanofluidic ion flux, Annu. Rev. Fluid Mech. 44, 401 (2012).

[7] V. S. Pham, Z. Li, K. M. Lim, J. K. White, and J. Han, Direct numerical simulation of electroconvective instability and hysteretic current-voltage response of a permselective membrane, Phys. Rev. E 86, 046310 (2012).

[8] I. Rubinstein and B. Zaltzman, Equilibrium Electroconvective Instability, Phys. Rev. Lett. 114, 114502 (2015).

[9] I. Rubinstein and B. Zaltzman, Equilibrium electro-osmotic instability in concentration polarization at a perfectly charge-selective interface, Phys. Rev. Fluids 2, 093702 (2017).

[10] A. Mani and K. M. Wang, Electroconvection near electrochemical interfaces: Experiments, modeling, and computation, Annu. Rev. Fluid Mech. 52, 509 (2020).

[11] W. H. Smyrl and J. Newman, Double layer structure at the limiting current, Trans. Faraday Soc. 63, 207 (1967).

[12] I. Rubinstein and L. Shtilman, Voltage against current curves of cation exchange membranes, J. Chem. Soc., Faraday Trans. II 75, 231 (1979).

[13] A. V. Listovnichy, Passage of currents higher than the limiting one through the electrode-electrolyte solution system, Sov. Electrochem. 25, 1651 (1989).
[14] V. V. Nikonenko, V. I. Zabolotsky, and N. P. Gnusin, Electric transport of ions through diffusion layers with impaired electroneutrality, Sov. Electrochem. 25, 262 (1989).

[15] M. Z. Bazant, K. T. Chu, and B. J. Bayly, Current-voltage relations for electrochemical thin films, SIAM J. Appl. Math. 65, 1463 (2005).

[16] I. Rubinstein and B. Zaltzman, Dynamics of extended space charge in concentration polarization, Phys. Rev. E 81, 061502 (2010).

[17] R. S. Aleksandrov, A. P. Grigin, and A. D. Davydov, Numerical study of Rayleigh-Benard instability of a binary electrolyte in a cell with plane parallel electrodes taking into account the space charge, Russ. J. Electrochem. 39, 370 (2003).

[18] E. Karatay, M. B. Andersen, M. Wessling, and A. Mani, Coupling Between Buoyancy Forces and Electroconvective Instability Near Ion-Selective Surfaces, Phys. Rev. Lett. 116, 194501 (2016).

[19] J. C. de Valença, R. M. Wagterveld, R. G. H. Lammertink, and P. A. Tsai1, Dynamics of microvortices induced by ion concentration polarization, Phys. Rev. E 92, 031003(R) (2015).

[20] J. C. de Valença, A. Kurniawan, R. M. Wagterveld, J. A Wood, and R. G. H. Lammertink, Influence of Rayleigh-Bénard convection on electrokinetic instability in overlimiting current conditions, Phys. Rev. Fluids 2, 033701 (2017).

[21] E. A. Demekhin, S. Amiroudine, G. S. Ganchenko, and N. Y Khasmatulina, Thermoelectroconvection near charge-selective surfaces, Phys. Rev. E 91, 063006 (2015).

[22] F. Maletzki, H. W. Rosler, and E. Staude, Ion transfer across electrodialysis membranes in the overlimiting current range: stationary voltage current characteristics and current noise power spectra under different conditions of free convection, J. Membr. Sci. 71, 105 (1992).

[23] V. V. Nikonenko, A. V. Kovalenko, M. K. Urtenov, N. D. Pismenskaya, J. Han, Ph. Sistat, and G. Pourcelly, Desalination at overlimiting currents: State-of-the-art and perspectives, Desalination 342, 85 (2014).

[24] I. Rubinstein, E. Shtaude, and O. Kedem, Role of the membrane surface in concentration polarization at ion-exchange membrane, Desalination 69, 101 (1988).

[25] S. S. Dukhin and B. V. Derjaguin, Electrophoresis (Nauka, Moscow, 1976). 